\title{
Australian bat lyssavirus: implications for public health
}

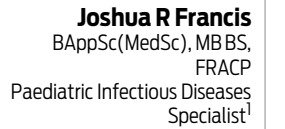

Bradley J McCall

MBBS, MPH, FAFPHM Public Health Physician ${ }^{2}$

Penny Hutchinson

MBBS, MPH, FAFPHM Public Health Physician and Director $^{3}$

Jodie Powell RN, PGCertInfectControl, BN Head of Infection Control ${ }^{4}$

Vikram L Vaska FRACP, FRCPA Microbiologist and Paediatric Infectious Diseases Specialist ${ }^{5}$

Clare Nourse MBBCh, FRACP, MD Director of Paediatric Infection Management Service ${ }^{4}$

1Department of Paediatrics, Royal Darwin Hospital, Darwin, NT.

2 Metro South Public Health Unit, Queensland Health, Brisbane, QLD.

3 Darling Downs Public Health Unit, Queensland Health,

Toowoomba, QLD.

4 Mater Health Services, Brisbane, QLD.

5 Mater Pathology, Brisbane, QLD.

josh.francis@nt.gov.au

doi: 10.5694/mjal3.00261 ustralian bat lyssavirus (ABLV) is genotype 7 of the 12 known lyssaviruses and is closely phylogenetically related to rabies (genotype 1$).{ }^{1}$ Although indigenous cases of rabies have never been identified in Australia, low-level ABLV endemicity has been found in Australian bats. Two variants have been identified: pteropid-ABLV, which has been found in all four Australian flying fox species (Megachiroptera), and YBST-ABLV, which has been identified in yellow-bellied sheathtail bats, a species of microbats (Microchiroptera). ${ }^{2}$ Both variants have been implicated in fatal human infection, albeit rarely. ${ }^{3-5}$ The third confirmed human case of ABLV infection, and the first in a child, was recently reported. ${ }^{5}$ Here, we outline the public health considerations of this emerging infection.

\section{Clinical aspects}

All three reported cases of human ABLV infection had clinical courses consistent with what is known of encephalitic (furious) rabies. Encephalitic rabies presents between several weeks and many years after exposure. It is usually characterised by progressive cerebral and autonomic dysfunction, preceded by a short, non-specific prodrome. Hydrophobic and aerophobic spasms are pathognomonic. The patient may initially be calm and cooperative, with interrupting and escalating episodes of confusion, agitation and hyperactivity. Deterioration, coma and death are inevitable. ${ }^{1,6}$

Diagnosis of lyssavirus infection is difficult and frequently delayed. Neuroimaging and cerebrospinal fluid (CSF) analysis may initially yield normal results, and serum and CSF lyssavirus antibody test results are frequently negative. ${ }^{1,6}$ ABLV can be identified using rabies reverse-transcription polymerase chain reaction assays, for which nuchal skin biopsy samples provide the greatest sensitivity, compared with saliva and CSF assays. Repeat testing is associated with improved sensitivity and is often necessary. ${ }^{2,7}$ Experimental treatment regimens, notably the Milwaukee Protocol, may be considered, but none have proven consistently effective and palliation is appropriate management in most cases. ${ }^{8-10}$

\section{Public health management of human exposure to bats}

Prevalence of ABLV is low $(<1 \%)$ in the healthy, wild bat population but is between $5 \%$ and $10 \%$ in sick, injured or orphaned bats. ${ }^{11,12}$ Since 1996, ABLV has been diagnosed in bats in New South Wales, the Northern

\section{Summary \\ Australian bat lyssavirus (ABLV) infection in humans is rare but fatal, with no proven effective therapy. \\ ABLV infection can be prevented by administration of a post-exposure prophylaxis regimen of human rabies immunoglobulin and rabies vaccine. \\ All Australian bats (flying foxes and microbats) should be considered to be carrying ABLV unless proven otherwise. \\ Any bat-related injury (bite, scratch or mucosal exposure to bat saliva or neural tissue) should be notified immediately to the relevant public health unit - no matter how small the injury or how long ago it occurred. \\ Human-to-human transmission of ABLV has not been reported but is theoretically possible. \\ Standard infection control precautions should be employed when managing patients with suspected or confirmed ABLV infection.}

Territory, Queensland, South Australia, Victoria and Western Australia. ${ }^{13}$ Transmission to humans occurs through direct inoculation of saliva from an infected primary host, typically through a bite or scratch. Wounds caused by bats may seem innocuous, but any potential abrasion or penetration of skin or mucous membranes should be taken seriously. Contact of mucous membranes or broken skin with the saliva or neural tissues of a bat in Australia constitutes potential exposure to ABLV. There is no evidence that transmission occurs through contact with bat urine, faeces or blood. In the United States, batvariant rabies has been diagnosed in people without a clear history of bat-inflicted injury, reinforcing the need for a cautious approach in the setting of potential exposures. ${ }^{14}$ All potential exposures to ABLV should be notified immediately to local public health authorities. ${ }^{15}$

The essential components of managing potential ABLV exposure include local wound treatment and initiation of post-exposure prophylaxis (PEP). Thorough wound cleansing reduces the risk of rabies transmission in animal models and is likely to be beneficial in preventing ABLV infection. Wounds should be irrigated with soap and water for at least 5 minutes, followed by application of a virucidal antiseptic solution such as povidone-iodine or alcohol. Unlike dog bites, bat bites or scratches result in small wounds that rarely require suturing. In the event that it is indicated, suturing of the wound should be delayed until after administration of human rabies immunoglobulin (HRIG) ${ }^{15}$

PEP, using a combination of multiple doses of rabies vaccine and HRIG, is safe and effective in preventing 
1 Bats and humans - key messages for the public

- All Australian bats (flying foxes and microbats) should be considered to be carrying Australian bat lyssavirus unless proven otherwise.

Bats should only be handled by trained and vaccinated wildlife handlers.

- Any bat-related injury (bite, scratch or mucosal exposure to bat saliva or neural tissue) should be reported immediately to a doctor - no matter how small the injury or how long ago it occurred.

- All wounds should be irrigated with water and soap for at least 5 minutes, as soon as possible after the injury occurs.

rabies in exposed individuals. ${ }^{16}$ Rabies vaccine is also protective against ABLV and has been effectively used for pre-exposure vaccination and for PEP. ${ }^{15} \mathrm{PEP}$ should be commenced as soon as a potential exposure is identified. If the implicated animal can be tested and has negative test results for lyssavirus, the PEP regimen may be discontinued. ${ }^{15,17}$ Previously unvaccinated individuals should receive intramuscular rabies vaccine on Days $0,3,7$ and 14. HRIG should be administered subcutaneously at the site of any wound as much as possible (up to the entire required dose), with the remainder of the dose (often up to half the required dose) given intramuscularly. Individuals who have previously been adequately vaccinated against rabies do not require HRIG and should be given a modified course of two doses of rabies vaccine. ${ }^{15}$

Given the potential for prolonged incubation periods, PEP should be offered to any person who has any history (no matter how distant) of a bite, scratch or mucous membrane or broken skin contact with the saliva or neural tissue of a potentially infected animal, including any bat in Australia or overseas. ${ }^{15}$

Ensuring the public (Box 1) and clinicians (Box 2) are aware of the importance of even minor exposures to bats remains an ongoing concern, given the effectiveness of PEP in preventing ABLV and the lack of therapeutic options after onset of active disease. A recent survey conducted in Queensland found relatively high levels of community awareness that bats pose some risks to human health. ${ }^{18}$ However, understanding of the full significance of exposure to bats is variable and appears heavily influenced by media reporting. Notification of potential exposures in Queensland peaked after the public reporting of previous ABLV cases, but subsequently returned to baseline. ${ }^{19}$ The bat encounter that preceded the most recent case of ABLV infection was not brought to medical attention at the time. ${ }^{5}$ In a 3-month period after public reporting of the case, 169 reports of potential ABLV exposure were reported to Queensland public health units, compared with an average of 13 per month in the preceding 2 calendar years (unpublished data, Communicable Diseases Unit, Queensland Health). This increase reflects greater public and clinician awareness of the risk of potential exposure following publicity of the case.

\section{Public health management of exposure to people with lyssavirus infection}

Human-to-human transmission of rabies and other lyssaviruses is considered theoretically possible, but has not been proven to occur. ${ }^{20}$ The only two reported cases of rabies thought to have resulted from direct human salivary contact were not substantiated by laboratory investigations. ${ }^{21}$ Rabies transmission via transplantation of infected organs has been well documented. Acquisition in the laboratory has also been reported, through direct inhalation of concentrated virus. Aerosolisation of virus contained in human tissue or secretions has not been proven to occur. ${ }^{20}$ Guidelines published by the Communicable Diseases Network Australia (CDNA) and the US Centers for Disease Control and Prevention recommend PEP for contacts who have had exposure to the saliva or neural tissue of an infected person across mucous membranes or broken skin. ${ }^{15,20}$

The routine delivery of health care to a patient with lyssavirus infection should not ordinarily pose any risk of transmission. Standard precautions provide adequate infection control, with emphasis on the use of eye protection, gowns, gloves and masks during procedures where contact with patient secretions is likely (eg, suctioning and intubation). ${ }^{20}$

After the most recent diagnosis of ABLV infection, which occurred at our institution in 2013, infection control and public health unit staff coordinated the identification and assessment of all potential contacts in the hospital and community. PEP was offered on the basis of percutaneous or permucosal exposure to the infected patient's saliva or neural tissue (CSF). Of 170 health care workers screened by infection control staff, 61 (35.9\%) were referred for medical review by the infectious diseases team because of potential or uncertain exposure, or anxiety. Fifteen (8.8\%) commenced PEP: eight met the CDNA criteria ${ }^{15}$ and the other seven specifically requested PEP in the setting of an ill defined exposure. Clinically significant exposures included contamination of broken skin and mucosal surfaces with respiratory secretions during airway management procedures, ${ }^{7,8}$ emphasising the importance of compliance with the standard precautions that could have prevented this exposure. One penetrating injury was sustained during initial assessment. The seven staff members who requested PEP had been involved in airway management procedures but could not recollect a specific incident of exposure to saliva or CSF.

Five household members were also considered to have had clinically significant exposure, resulting in a total of 20 people who were administered PEP. Two who had been previously vaccinated against rabies received a course of two doses of rabies vaccine only; another two declined HRIG and received vaccine only; and 16 completed a full course of PEP with rabies vaccine and HRIG. One PEP recipient experienced minor adverse effects after vaccination, but was able to complete the course. No secondary cases of ABLV have been identified.

\footnotetext{
2 Bats and humans - key messages for the clinician
}

Any bat-related injury (bite, scratch or mucosal exposure to bat saliva or neural tissue), including those inflicted by a microbat (insectivorous bat), should be notified immediately to your local public health unit - no matter how small the injury or how long ago it occurred.

All wounds should be irrigated with water and soap for at least 5 minutes, as soon as possible after the injury occurs, followed by application of povidone-iodine or alcohol.

If a clinically significant potential exposure is identified, in liaison with your local public health unit, post-exposure prophylaxis, using human rabies immunoglobulin and rabies vaccine as indicated, should be commenced.

Standard infection control precautions, including the use of eye protection, gowns, gloves and masks during aerosol-generating procedures, should be employed when managing patients with Australian bat lyssavirus infection. 
PEP is used more liberally in other parts of the world, with reports of large numbers of health care workers receiving prophylaxis on the basis of "possible" as well as "proven" contact. ${ }^{22,23}$ The approach taken in the US and Australia results in less administration of PEP, but in our opinion is scientifically valid and appropriately cautious. Although potential contacts without a defined exposure are not routinely given PEP, no cases of lyssavirus transmission to a health care worker in the context of direct patient care have been reported.

\section{Other potential hosts}

Bats are the primary host for ABLV. Spread to other mammalian species through exposure to infected bats is possible but has rarely been reported. Experimental inoculation of domestic cats and dogs with ABLV resulted in seroconversion and mild behavioural changes, but no evidence of clinical disease or ongoing viral replication. ${ }^{24}$ There have been no documented cases of proven ABLV infection in dogs. In 2013, two horses in south-east Queensland with clinical disease were found to have ABLV infection - the first confirmed cases of ABLV in animals other than bats or humans. ${ }^{25}$ The risk of secondary transmission of ABLV to humans from animals other than bats is likely to be very low. In Australia, in addition to being recommended for those with exposure to bats (as above), PEP should also be considered in the setting of potential exposure to any other animal with proven ABLV infection.

\section{Conclusion}

Human infection with ABLV is rare but devastating. Health care workers and the Australian public should be well informed of the risk of transmission from microbats and flying foxes in Australia, and educated about the importance and reliability of PEP in preventing disease. The risk posed to contacts of people with ABLV infection is unknown but probably very low in most circumstances. In spite of this, the expectation that ABLV will continue to be universally fatal in humans warrants a cautious approach, including the administration of PEP when clinically significant contact with infected material occurs. The number of health care workers given PEP after the recent diagnosis of ABLV in a patient illustrates the importance of strict compliance with standard precautions in the care of hospital inpatients.

At the time of the most recent case and during the resultant contact tracing, licensed rabies vaccine and HRIG were available in sufficient supply. Subsequently, greater local demand due to increased awareness of ABLV has combined with worldwide shortages of both vaccine and HRIG to pose significant challenges for the timely delivery of PEP when it is indicated. Notwithstanding this, CDNA guidelines for managing potential lyssavirus exposures ${ }^{15}$ remain appropriate in the Australian setting.

Acknowledgements: We acknowledge the staff from many units who contributed to the clinical and public health management of the most recent case of human ABLV infection, including the Mater Children's paediatric intensive care unit, Mater Health Services infection control and staff health units, Metro South Public Health Unit,
Queensland Health Communicable Diseases Unit and Queensland Health Forensic and Scientific Services.

Competing interests: No relevant disclosures.

Provenance: Not commissioned; externally peer reviewed.

1 Warrell MJ, Warrell DA. Rabies and other lyssavirus diseases. Lancet 2004; 363: 959-969.

2 Guyatt KJ, Twin J, Davis P, et al. A molecular epidemiological study of Australian bat lyssavirus. J Gen Virol 2003; 84 (Pt 2): 485-496.

3 Samaratunga $H$, Searle JW, Hudson N. Non-rabies Lyssavirus human encephalitis from fruit bats: Australian bat Lyssavirus (pteropid Lyssavirus) infection. Neuropathol Appl Neurobiol 1998; 24: 331-335.

4 Hanna JN, Carney IK, Smith GA, et al. Australian bat lyssavirus infection: a second human case, with a long incubation period. Med J Aust 2000; 172: 597-599.

5 Francis JR, Nourse C, Vaska VL, et al. Australian Bat Lyssavirus in a child: the first reported case. Pediatrics 2014; 133: e1063-el067. doi: 10.1542/ peds.2013-1782.

6 Hemachudha T, Laothamatas J, Rupprecht CE. Human rabies: a disease of complex neuropathogenetic mechanisms and diagnostic challenges. Lancet Neurol 2002; 1: 101-109.

7 Dacheux L, Reynes JM, Buchy P, et al. A reliable diagnosis of human rabies based on analysis of skin biopsy specimens. Clin Infect Dis 2008; 47: 1410-1417.

8 Jackson AC. Current and future approaches to the therapy of human rabies. Antiviral Res 2013; 99: 61-67.

9 Jackson AC, Warrell MJ, Rupprecht CE, et al. Management of rabies in humans. Clin Infect Dis 2003; 36: 60-63.

10 Willoughby RE Jr, Tieves KS, Hoffman GM, et al. Survival after treatment of rabies with induction of coma. NEngl J Med 2005; 352: 2508-2514.

11 McCall BJ, Epstein JH, Neill AS, et al. Potential exposure to Australian bat lyssavirus, Queensland, 1996-1999. Emerg Infect Dis 2000; 6: 259-264.

12 Warrilow D, Harrower B, Smith IL, et al. Public health surveillance for Australian bat lyssavirus in Queensland, Australia, 2000-2001. Emerg Infect Dis 2003; 9: 262-264.

13 Grillo T, Cox-Witton K, Post L. Australian Wildlife Health Network. Animal Health Surveillance Quarterly Report 2012; 17: 5-6. http://www.sciquest. org.nz/elibrary/download/81605/Australian_Wildlife_Health_Network. pdf? (accessed Jul 2014).

14 De Serres G, Dallaire F, Côte M, Skowronski DM. Bat rabies in the United States and Canada from 1950 through 2007: human cases with and without bat contact. Clin Infect Dis 2008; 46: 1329-1337.

15 Communicable Diseases Network Australia. Rabies virus and other lyssavirus (including Australian Bat Lyssavirus) exposures and infections: CDNA national guidelines for public health units. http://www.health.gov. au/internet/main/publishing.nsf/Content/cdna-song-abvl-rabies.htm (accessed May 2013).

16 Evans JS, Horton DL, Easton AJ, et al. Rabies virus vaccines: is there a need for a pan-lyssavirus vaccine? Vaccine 2012; 30: 7447-7454.

17 Ewald B, Durrheim D. Australian Bat Lyssavirus: examination of postexposure treatment in NSW. NS W Public Health Bull 2008; 19: 104-107.

18 Young MK, El Saadi D, McCall BJ. Preventing Australian bat lyssavirus: community knowledge and risk perception of bats in South East Queensland. Vector Borne Zoonotic Dis 2014; 14: 284-290.

19 Young MK, McCall BJ. Trends in potential exposure to Australian bat lyssavirus in South East Queensland, 1996 to 2003. Commun Dis Intell Q Rep 2004; 28: 258-260.

20 Manning SE, Rupprecht CE, Fishbein D, et al. Human rabies prevention United States, 2008: recommendations of the Advisory Committee on Immunization Practices. MMWR Recomm Rep 2008; 57: 1-28.

21 Fekadu M, Endeshaw T, Alemu W, et al. Possible human-to-human transmission of rabies in Ethiopia. Ethiop Med J 1996; 34: 123-127.

22 Mattner F, Bitz F, Goedecke M, et al. Adverse effects of rabies pre- and postexposure prophylaxis in 290 health-care-workers exposed to a rabies infected organ donor or transplant recipients. Infection 2007; 35: 219-224.

23 Mattner F, Henke-Gendo C, Martens A, et al. Risk of rabies infection and adverse effects of postexposure prophylaxis in healthcare workers and other patient contacts exposed to a rabies virus-infected lung transplant recipient. Infect Control Hosp Epidemiol 2007; 28: 513-518.

24 McColl KA, Chamberlain T, Lunt RA, et al. Susceptibility of domestic dogs and cats to Australian bat lyssavirus (ABLV). Vet Microbiol 2007; 123: 15-25.

25 Queensland Government Department of Agriculture, Fisheries and Forestry. Australian bat lyssavirus overview. 2013. http://www.daff.qld.gov. au/animal-industries/animal-health-and-diseases/a-z-list/australianbat-lyssavirus/australian-bat-lyssavirus (accessed Dec 2013). 